\title{
Epithèse nasale implanto-portée en carcinologie : à propos d'un cas clinique
}

\section{Implant-supported epithesis in oncology: a case report}

\author{
ANNE-GAËLLE BODARD*, JULIE BÉMER*, CHRISTIAN BUGEAUD*, RENÉ GOURMET*
}

\section{RÉSUMÉ}

Le cas d'une femme de 60 ans ayant bénéficié de l'exérèse carcinologique du seuil narinaire droit et du bord libre de la cloison nasale est rapporté. Après la chirurgie reconstructrice, deux implants endo-osseux (Ankylos ${ }^{\circledR}$ EO $3.5 \times 5 \mathrm{~mm}$ ) ont été mis en place. Après ostéointégration, un épithèse définitive a été réalisée sur pilier aimanté.

Le choix des implants endo-osseux se justifie par le volume osseux disponible et le recul clinique pour ce type d'implants. L'ancrage aimanté a été préféré pour son faible encombrement et sa simplicité. Les épithèses implanto-portées offrent une solution, tant fonctionnelle qu'esthétique, fiable et prédictible. Med Buccale Chir Buccale 2007; 13: 93-96.

mots clés: nez, épithèse, implants extra-oraux

médecine buccale chirurgie buccale

VOL. $13, N^{\circ} 2$ 2007

page 93

\section{SUMMARY}

The case of a 60-years old woman is reported. An exeresis of the base of the nose was done. Reconstructive surgery reduced the loss of substance but did not allow total repair. Two endosseous implants (Ankylos ${ }^{\oplus O} 3.5 \times 5$ $\mathrm{mm}$ ) were placed. The permanent epithesis was done after osteointegration on a $6 \mathrm{~mm}$ magnetic abutment. The epithesis is a complement of the reconstructive surgery. The choice of endosseous implants is justified by osseous volume and clinical hindsight of these sort of implants. Magnetic anchorage is chosen for its size, its easy cleaning and setting up. Implant-retained prostheses offer a functional and aesthetic solution. Med Buccale Chir Buccale 2007; 13: 93-96.

key words: nose, epithesis, extraoral implants

* Service de Chirurgie, Centre Léon Bérard, 28 rue Laënnec, 69373 Lyon Cedex 08

** Facial Laboratoire, 15 rue du Château, 69008 Lyon

Demande de tirés à part:

Anne-Gaëlle Bodard Unité d'Odontologie Service de Chirurgie Centre Léon Bérard 28 rue Laënnec 69373 Lyon Cedex 08 bodard@lyon.fnlcc.fr 
médecine

buccale

chirurgie

buccale

VOL. $13, \mathrm{~N}^{\circ} 2$

2007

page 94
La chirurgie reconstructrice constitue, dans la plupart des cas, une solution suffisante pour les pertes de substance faciales. Elle n'est pas toujours applicable en carcinologie, soit pour des raisons loco-régionales (tissus cicatriciels ou irradiés) ou générales (état général du patient) [1]. De plus, les résultats sont difficilement prévisibles et de nombreux patients hésitent à entreprendre de multiples interventions pour un résultat esthétique incertain. C'est le cas pour les pertes de substance nasales car l'anatomie du nez est complexe à reproduire et nécessite des greffes osseuses et cartilagineuses multiples en plus des lambeaux régionaux ou microanastomosés. Les épithèses présentent un avantage car elles permettent une réhabilitation rapide et sûre, en restaurant à la fois l'aspect esthétique et fonctionnel. Les moyens d'ancrage de l'épitho-prothèse sont multiples : lunettes, colles et ancrage osseux. Les travaux de Brånemark, Tjellström et Albreksson [2] ont permis de proposer des moyens fiables de fixation osseuse pour les épithèses faciales sur implants.

\section{OBSERVATION}

Le cas clinique présenté est celui d'une femme âgée de 60 ans. En juillet 1999, l'exérèse in sano d'un adénocarcinome sudoral très infiltrant du seuil narinaire droit et de la région sous-nasale est réalisée.

Figure 1 : Vue peropératoire : mise en place des implants dans le maxillaire antérieur

Peroperative view: placement of the implants in the maxilla

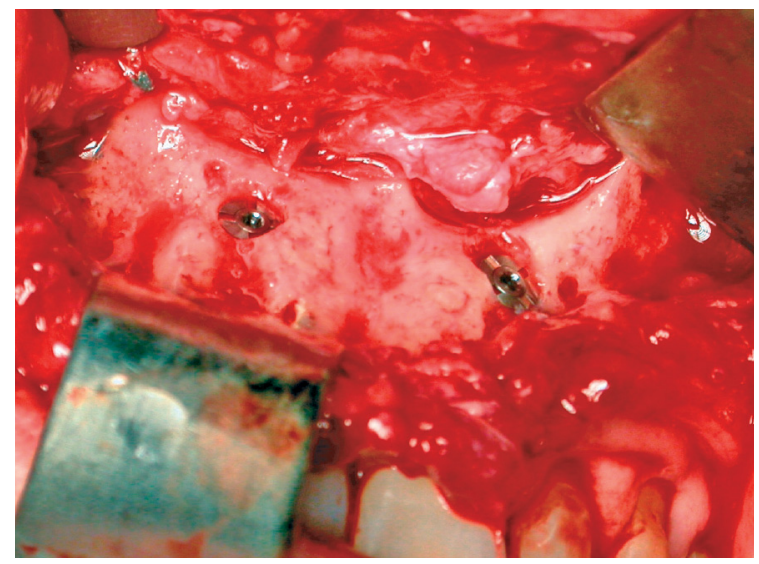

Figure 2 : Pilier translabial : noter l'absence d'inflammation tissulaire

Translabial abutment: notice the non inflammatory soft tissues

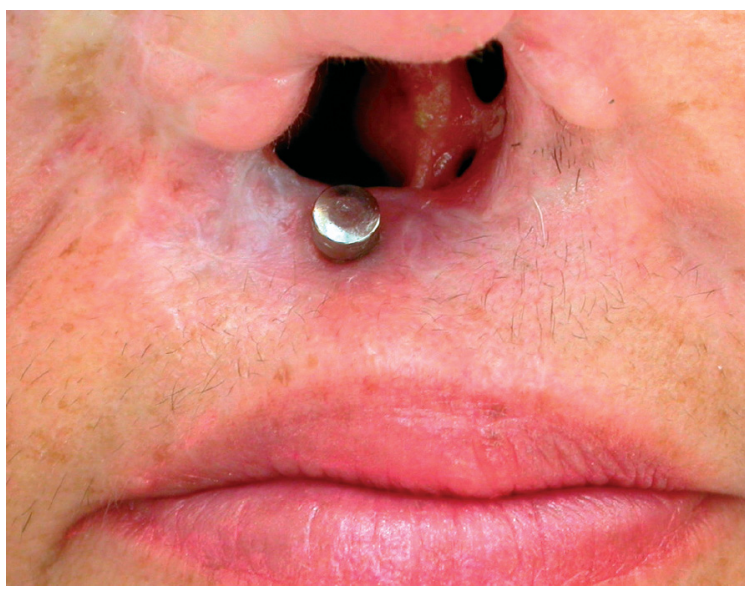

Figure 3 : Résultat final : épithèse en place; vue de face

Final result: facial view with epithesis

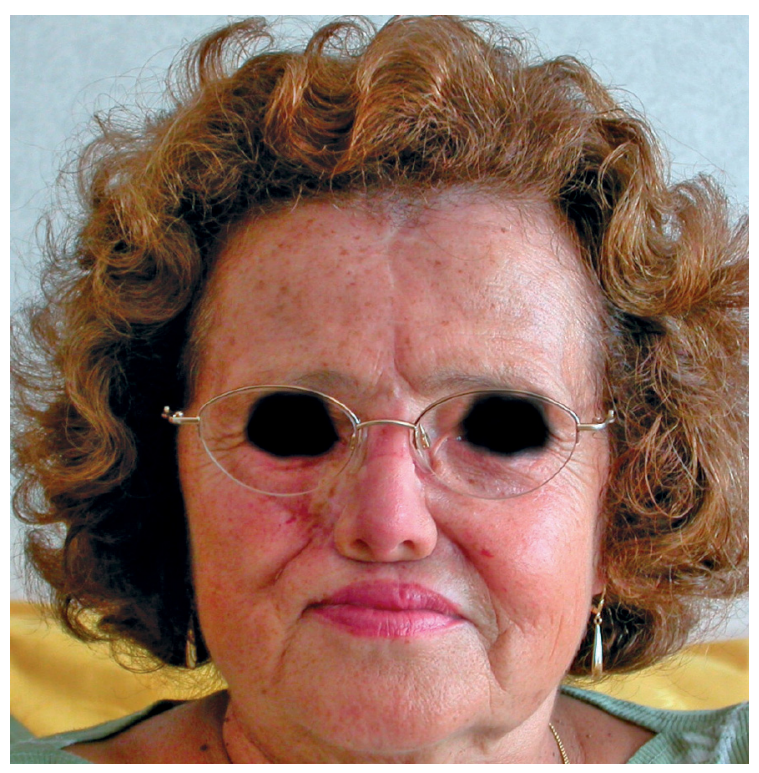

La radiothérapie n'est pas proposée. Une série d'interventions reconstructrices par lambeaux jugaux d'avancement est conduite, permettant la fermeture partielle de la perte de substance. Une première épithèse collée est réalisée fin 2001. En mars 2004, devant la nécessité de réfection de l'épithèse, l'alternative implantaire est proposée.

Le bilan clinique se compose de l'examen des tissus environnants : leur aspect, leur épaisseur, leur 
souplesse, leur sensibilité et leur motricité résiduelle sont évalués. L'état dentaire est plus particulièrement étudié au maxillaire : la patiente est totalement dentée dans la région antérieure ; seules manquent au maxillaire les molaires droites. L'orthopantomographie objective l'absence de tout foyer infectieux dentaire et l'examen tomodensitométrique montre un volume osseux disponible de 4 à $6 \mathrm{~mm}$ dans la région paramédiane ; la présence de toutes les dents dans la région antérieure complique l'implantation qui devra éviter les racines dentaires.

Une maquette guide en silicone est réalisée : elle préfigure le volume et les limites de la future épithèse, l'emplacement des orifices narinaires et l'émergence implantaire idéale.

En décembre 2004, sous anesthésie générale, deux implants Ankylos ${ }^{\oplus}$ extra-oraux (EO) de $3.5 \times 5 \mathrm{~mm}$ sont mis en place dans la région sous-nasale du maxillaire. En avril 2005, le second temps opératoire consiste à la mise en place d'un pilier de cicatrisation translabial de $6 \mathrm{~mm}$. Un seul implant est exploité, le second maintenu enfoui. Un pilier aimanté Ankylos ${ }^{\circledR}$ de $6 \mathrm{~mm}$ est mis en place sous anesthésie locale 15 jours après le second temps chirurgical. Des conseils de maintenance et d'hygiène sont donnés à la patiente. L'épithèse définitive est ensuite réalisée en silicone.

\section{DISCUSSION}

Le cas clinique présenté souligne la complémentarité entre la chirurgie reconstructrice et la prothèse faciale : en effet, la patiente a bénéficié de plusieurs interventions de chirurgie plastique qui ont permis la réduction de la perte de substance ; elles n'ont pas été suffisantes pour obtenir une reconstitution complète du nez. L'épithèse a permis de pallier aux limites de la chirurgie, tout en offrant un résultat esthétique et fonctionnel prévisible. La perte de substance résiduelle était peu importantes (pointe du nez, bord libre de la cloison nasale et région sous-nasale) et bordée par des tissus mous cicatriciels provenant de lambeaux de proximité. Seule la limite inférieure de la perte de substance repose sur une structure osseuse, correspondant au maxillaire. Pour Jensen ${ }^{[3]}$, le plancher des fosses nasales consti- tue le site d'implantation le plus fiable pour l'ancrage des épithèses nasales. II s'agit d'un site de type alpha (plus de $6 \mathrm{~mm}$ d'os) ou bêta (de 4 à $6 \mathrm{~mm}$ d'os), autorisant la mise en place d'implants-vis de faible longueur. Jensen conseille leur mise en place selon un angle de $60^{\circ}$ par rapport à l'horizontale. Elle doit être guidée par une maquette chirurgicale qui permet de visualiser les limites de la future épithèse et les orifices narinaires, et de déterminer l'émergence implantaire idéale. Ceci est d'autant plus important pour un bon résultat esthétique que le volume de l'épithèse est réduit. Dans le cas présenté, l'émergence implantaire devait éviter de se trouver en regard des orifices narinaires, rester en deçà des limites de l'épithèse et l'axe des implants respecter les racines dentaires. L'indication de la solution implantaire reposait sur plusieurs éléments : absence de rétention anatomique, stabilité de l'épithèse, camouflage permanent de la perte de substance, possibilité de réaliser des bords plus esthétiques, mise en place d'une épithèse simplifiée. Le choix des implants a été dicté par le volume osseux d'une part et le recul clinique sur ce type d'implants d'autre part. Un implant n'a pas été utilisé car il n'était pas nécessaire pour la stabilité de l'épithèse : pour cette épithèse de faible dimension, soumise à peu de forces, l'appui sur les ailes du nez était suffisante pour assurer une bonne stabilité. Pour la rétention, un système magnétique a été choisi car il présente plusieurs avantages : faible encombrement, nettoyage aisé, facilité de mise en place de l'épithèse et possibilité de choisir des aimants de différentes forces. Celle-ci a été déterminée en fonction du poids de l'épithèse et de l'habileté de la patiente. La cicatrisation cutanée autour de l'émergence implantaire a été rapide et la patiente n'a présenté ni suppuration, ni suintement, ni érythème. L'absence de follicule pileux, la finesse des tissus et leur faible mobilité (tissus cicatriciels) ainsi que les soins d'hygiène réalisés par la patiente ont favorisé la tolérance de l'épithèse [4]. L'épithèse implanto-portée doit être une solution thérapeutique à proposer au patient ; elle est le fruit d'une collaboration étroite entre les chirurgiens, l'épithésiste et le patient. La qualité de vie se trouve nettement améliorée après mise en médecine buccale chirurgie buccale VOL. $13, \mathrm{~N}^{\circ} 2$ 2007 page 95 
place d'une épithèse implanto-portée [5] : la stabilité prothétique n'est pas perturbée par la transpiration, l'activité (notamment en milieu aqueux) ou les éternuements, et la mise en place de l'épithèse est facilitée. A l'avenir la conception et la

\section{RÉFÉRENCES}

1 - Labbé D, Benateau H, Compère JF, Sabin P. Implants extraoraux : indications et contre-indications. Rev Stom Chir Maxillofac $2001 ; 102$ : 239-42.

2 - Tjellström A. Osseointegrated systems and their application in the head and neck. Adv Otolaryngol Head Neck Surg 1989; $3: 39-47$.

3 - Jensen OT, Brownd C, Blaker J. Nasofacial prosthesis supported by osseointegrated implants. Int J Oral Maxillofac Impl $1992 ; 7$ : 203-11.

médecine
buccale chirurgie buccale

VOL. $13, \mathrm{~N}^{\circ} 2$ 2007

page 96
4 - Roumanas ED, Freymiller EC, Chang TL, Aghaloo T. Beumer J 3rd. Implant retained prostheses for facial defects : an up to 14-year follow up report on the survival rates of implants at UCLA. Int J Prosthod 2002 ; $15: 325-32$. réalisation guidée par ordinateur (planification de la chirurgie implantaire, réalisation de la prothèse) de ces épithèses implanto-portées seront rendues plus aisées par l'utilisation de modèles stéréolithographiques ${ }^{[6]}$.
5 - Chang TL, Garett N, Roumanas E, Beumer J 3rd. Treatment satisfaction with facial prosthesis. J Prosth Dent 2005 ; 94 : 275-80.

6 - Verdonck HW, Poukens J, Overveld HV, Rieidger D. Computer-assisted maxillofacial prosthodontics: a new treatment protocol. Int J Prodthod 2003 ; 16 : 326-8. 